\title{
Effects of Dietary Fermented Garlic on the Growth Performance, Relative Organ Weights, Intestinal Morphology, Cecal Microflora and Serum Characteristics of Broiler Chickens
}

-Author(s)

Lee KW'
Lee $K C^{\prime}$
Kim $\mathrm{GH}^{\prime}$
Kim JH'
Yeon JS'
Cho SB"
Chang BJ"'
Kim SK'

Department of Animal Science and Technology, College of Animal Bioscience and Technology, Konkuk University, 120 Neungdong-ro, Gwangjin-gu, Seoul 143701, Republic of Korea

Animal Resources Research Center, Konkuk University, 120 Neungdong-ro, Gwangjingu, Seoul, 143-701, Republic of Korea

III Department of Physiology and Anatomy, College of Veterinary Medicine, 120 Neungdong-ro, Gwangjin-gu, Seoul 143701, Republic of Korea

\section{-Mail Address}

Corresponding author e-mail address S. K. Kim

Department of Animal Science and Technology, College of Animal Bioscience and Technology, 120 Neungdong-ro, Gwangjin-gu, Seoul 143-701, South Korea

Tel: $\quad+82.2 .4503728$

Fax: $\quad+82.2 .4583728$

Email: sookikim@konkuk.ac.kr

\section{EKeywords}

Broiler chickens, Growth performance, Gut morphology, Leuconostoc citreum SK2556.

\section{ABSTRACT}

The present study was conducted to evaluate the effects of feeding broilers with garlic fermented by Leuconostoc citreum SK2556. A total of 250 male broiler chicks was randomly housed into 25 floor pens. Five dietary treatments with five replicates of 10 chicks each ( $n=50$ chicks/ treatment). A corn and soybean meal based diet was used as the control diet (NC). The experimental diets were formulated by mixing the basal diet either with antibiotics (10 ppm; PC) or fermented garlic (FG) at the concentrations of $0.1 \%(F G 1), 0.3 \%(F G 3)$ or $0.5 \%(F G 5)$ in diets. Daily weight gain, feed intake, and feed:gain ratio were not affected by any of the dietary treatments. Average daily gain on day 21 linearly increased $(p=0.024)$ with increasing FG levels. The relative weight of the bursa of Fabricius showed a progressive decline with increasing the FG levels. Jejunal villus height was not influenced by dietary treatments. Villus width linearly decreased as FG levels increased $(p=0.17)$. Jejunal crypt depth was significantly lower $(p<0.05)$ in the FG 1 and FG3 groups compared with the NC group. Villus height:crypt depth ratio linearly increased $(p=0.018)$ with increasing FG levels. The population of cecal microflora was not altered by dietary treatments. Broiler chickens fed the FG5 diet exhibited $(p<0.05)$ higher blood levels of total protein and cholesterol compared with those fed the NC diet. Collectively, the results show that dietary FG marginally affected growth performance, especially during the first days rearing, improved intestinal morphology, and altered blood characteristics of broiler chickens.

\section{INTRODUCTION}

Garlic (allium sativum) has long history of use as culinary or medicinal supplement. As garlic contains a sulfur volatile active component that has antibacterial, anti-inflammatory and antioxidant biological properties (Wilson and Demming-Adams, 2007), it has been explored as a potential alternative to antibiotics in poultry production. Nonetheless, inconsistent effects of garlic on the growth performance, egg production, gut physiology, nutrient digestibility, and lipid metabolism have been reported (Khan et al., 2012). Garlic inclusion levels widely varied in those studies, being added at levels as low as $0.125 \%$ to as high as $5 \%$ to broiler diets.

Several attempts have been recently made to enhance garlic functionality and stability using fermentation with specific bacteria strains which are resistant to garlic, and are able to ferment it. The bacterial strains commonly used for garlic fermentation are Lactobacillus plantarum, Bacillus subtilis, Weissella koreensis, and Leukonostoc mesenteroides. Garlic fermented with either W. koreensis or $L$. mesenteroides equally produced higher sulfur-related metabolites, such as 3-vinyl-[4H]-1,2,-dithiin, all ylmethyl trisulfide, diallyl disulfide, 
Lee KW, Kim GH, Kim JH, Yeon JS, Cho SB, Chang BS, Kim SK
Effects of Dietary Fermented Garlic on the Growth Performance, Relative Organ Weights, Intestinal Morphology, Cecal Microflora and Serum Characteristics of Broiler Chickens diallyl trisulfide, but lower alliin compared with fresh garlic (Yan et al., 2012; Hussain et al., 2014).

Recent feeding trials have been tested the effect of fermented garlic on pig and broiler performance (Ao et al., 2010, 2011; Kang et al., 2010; Wang et al., 2011; Yang et al., 2012; Yan \& Kim, 2013; Hossain et al., 2014, 2015; Chun et al., 2015). The addition of graded levels (e.g., dietary inclusion levels of 0.05 , $0.1,0.2 \%$ ) of $L$. mesenterodes-fermented garlic in the diets of broiler chickens increased body weight and feed intake, but did not affect feed efficiency, when compared with the control-diet fed chickens (Hossain et al., 2014, 2015). These results corroborated with the study of Kang et al. (2011), who used L. plantarum for garlic fermentation and added higher levels (1\%) to a broiler diet. In contrast with the studies of Kang et al. (2011) and Hossain et al. (2014, 2015), dietary W. koreensis-fermented garlic failed to affect the growth performance of broiler chickens, albeit it enhanced host immunity, retarded meat lipid oxidation, and lowered serum total cholesterol and triglyceride concentrations compared with the control chickens (Ao et al., 2011).

In order to further increase our understanding of the efficacy of fermented garlic, we tested whether garlic fermented by L. citreum SK2556, a novel patented strain isolated from an aged garlic product, could affect the growth performance of broiler chickens, and if indeed so, whether the FG-induced improvement in production performance was related to changes in intestinal morphology. To our knowledge, no studies on the effect of $F G$ on the intestinal morphology of broiler chickens have been conducted to date. It was reported that dietary non-fermented garlic exhibited antibacterial activity and improved the intestinal morphology of broiler chickens (Abdullah et al., 2010; Ham et al., 2010). Thus, the present study aimed at investigating the effects of the dietary supplementation of FG on the growth performance, intestinal morphology and microbiota, and serum characteristics of broiler chickens.

\section{MATERIALS AND METHODS}

\section{Preparation of the fermented garlic powder}

The peeled garlic bulbs used in this study were purchased from a local market and ground in a mill (Philips HR 2860, Netherlands) to obtain a homogenized garlic juice. The garlic juice was then mixed with a buffer $\left(\mathrm{K}_{2} \mathrm{HPO}_{4} 0.1 \mathrm{~g} / \mathrm{L}, \mathrm{MgCl}_{2} 0.01 \mathrm{~g} / \mathrm{L}\right.$, $\left.\mathrm{NaCl} 1 \mathrm{~g} / \mathrm{L}, \mathrm{CH}_{3} \mathrm{COONa} 1 \mathrm{~g} / \mathrm{L}\right)$ at the ratio of $30: 70$. The garlic and buffer mixture was sterilized by autoclaving at $121^{\circ} \mathrm{C}$ for $15 \mathrm{~min}$ and cooled to room temperature. The mixture was then inoculated with a starter culture of $1 \%$ Leuconostoc citreum SK2556 in a shaking incubator at $37^{\circ} \mathrm{C}$ for $24 \mathrm{~h}$. The $\mathrm{pH}$ of the fermented garlic was 4.47. The L. citreum SK2556 culture contained a $1 \times 10^{8} \mathrm{cfu} / \mathrm{mL}$. The $L$. citreum SK2556 strain used in this study was originally isolated from pickled garlic food, characterized and patented due to its ability to grow and ferment in medium containing garlic (Kim et al., 2012).

\section{Experimental design, animals and diets}

A total of 250 feather-sexed male broiler chicks (Ross 308) were purchased from a local hatchery. Upon arrival, birds were individually weighed and randomly distributed into 25 floor pens with rice husks as a bedding material.

Five dietary treatments with five replicates (10 chicks per replicate, $n=50$ chicks/treatment) were applied. Starter and finisher diets based on corn and soybean meal were formulated (Tables 1 and 2) and used as

Table 1 - Ingredients and composition of basal diets

\begin{tabular}{|c|c|c|}
\hline Item & $\begin{array}{c}\text { Starter } \\
(0-21 \text { days })\end{array}$ & $\begin{array}{c}\text { Grower } \\
\text { (22-35 days) }\end{array}$ \\
\hline \multicolumn{3}{|l|}{ Ingredient, \% } \\
\hline Corn & 52.80 & 56.65 \\
\hline Wheat Powder & 5.00 & 5.00 \\
\hline Fish meal & 2.00 & 2.00 \\
\hline Giblet powder & 2.00 & 3.00 \\
\hline Soybean meal & 23.60 & 21.00 \\
\hline Full-fat soybean (extruded) & 5.00 & 3.00 \\
\hline Limestone & 1.33 & 1.27 \\
\hline Salt & 0.24 & 0.24 \\
\hline Glucose & 1.00 & 1.00 \\
\hline Dicalcium phosphate & 1.00 & 1.00 \\
\hline Tallow & 4.90 & 4.90 \\
\hline Vitamin and mineral premix ${ }^{1}$ & 0.15 & 0.15 \\
\hline L-Lysine- $\mathrm{HCl}$ & 0.45 & 0.39 \\
\hline DL-Methionine & 0.31 & 0.25 \\
\hline L-Threonine & 0.07 & 0.05 \\
\hline Choline- $\mathrm{HCl}$ & 0.05 & 0.05 \\
\hline Maduramicin & 0.05 & 0.05 \\
\hline Total & 100.0 & 100.0 \\
\hline \multicolumn{3}{|l|}{ Calculated nutrient composition } \\
\hline Metabolizable energy, MJ/kg & 13.7 & 13.1 \\
\hline Crude protein, \% & 21.04 & 20.70 \\
\hline Crude fat, \% & 8.59 & 8.43 \\
\hline Calcium, \% & 0.92 & 0.93 \\
\hline Available phosphorus, \% & 0.32 & 0.31 \\
\hline
\end{tabular}

'Vitamin and mineral premix contains followings per $\mathrm{kg}$ of diet: vitamin $\mathrm{A}$, 10,000,000IU; vitamin $\mathrm{D}_{3^{\prime}} 5,000,000 \mathrm{IU}$; vitamin $\mathrm{E}, 20,000 \mathrm{IU}$; vitamin $\mathrm{K}_{3^{\prime}}, 3000 \mathrm{mg}$; vitamin $B_{1}, 2000 \mathrm{mg}$; vitamin $B_{2}, 6000 \mathrm{mg}$; vitamin $B_{6}, 3000 \mathrm{mg}$; vitamin $B_{12}, 16 \mathrm{mg}$; niacin, 50,000mg; Ca-pantothenate, 13,000mg; folic acid, 13,000mg; Cu, 5000mg; I, 1250mg; Mn, 110,000mg; Zn, 100,000mg; Se, 300mg; Fe, 40,000mg; Co, 5000mg. 
Lee KW, Kim GH, Kim JH, Yeon JS, Cho SB, Chang BS, Kim SK
Effects of Dietary Fermented Garlic on the Growth Performance, Relative Organ Weights, Intestinal Morphology, Cecal Microflora and Serum Characteristics of Broiler Chickens non-medicated control diets (negative control, NC). The experimental diets were formulated by mixing the basal diet with either 10 ppm avilamycin (positive control, PC) or three levels of fermented garlic at the concentrations of $0.1 \%$ (FG1), $0.3 \%$ (FG3), or $0.5 \%$ (FG5). All diets contained maduramicin $(5 \mathrm{mg} / \mathrm{kg}$ of diet) as a coccidiostat. Diet and water were provided on an ad-libitum basis. The initial room temperature was set at $33^{\circ} \mathrm{C}$, gradually decreased to reach $25^{\circ} \mathrm{C}$ at 15 days of age and kept constant thereafter (Kang and Kim, 2016). All animal care procedures were approved by Institutional Animal Care and Use Committee in Konkuk University.

\section{Measurements}

Body weight and feed intake were measured by pen on a weekly basis and used to calculate feed:gain ratio. On day 35, three birds per pen were randomly selected for blood sampling after euthanasia. Immediately after blood sampling, the liver, spleen, and bursa of Fabricius were excised, weighed and expressed as relative weight in grams per $100 \mathrm{~g}$ of body weight. For the morphological determination of the villi, 1-cmlong sections from mid-jejunum and mid-ileum were sampled. Cecal content was sampled and kept on ice until measurement of microflora on a same day of the sampling.

\section{Intestinal morphology}

Intestinal samples were fixed in $10 \%$ phosphatebuffered formalin for a minimum of $48 \mathrm{~h}$, and $4.0 \mu \mathrm{m}$ sections were prepared as described elsewhere (Kim et al., 2015). The sections were stained with standard hematoxylin-eosin solution and villus height, villus width (mid-point), and crypt depth were measured at $100 x$ magnification under light microscopy. Villus surface area was calculated using the formula $=(2 \pi) \times$ $(\mathrm{VW} / 2) \times(\mathrm{VL})$, where $\mathrm{VW}=$ villus width and $\mathrm{VL}=$ villus length (Sakamoto et al., 2000). Twenty well-oriented villi per bird were measured and averaged for each bird.

\section{Enumeration of the cecal microflora}

Approximately $1 \mathrm{~g}$ of cecal contents were mixed with 9 volumes of sterilized ice-cold saline solution $(\mathrm{w} / \mathrm{v})$, vortexed, and serially diluted. A volume of 100 $\mu \mathrm{L}$ of cecal suspension was plated on the respective agar plates and incubated at $37^{\circ} \mathrm{C}$ for $48 \mathrm{~h}$. Colony was read and expressed as colony forming unit (cfu) per gram of cecal contents. The media used were MRS (Difco, USA), EMB (BBL TM Eosin Methylene Blue Agar,
USA), and SS-agar (BBLTM Salmonella Shigella Agar, USA). No colonies were detected in SS-agar. Colonies in MRS and EMB agars were selected and confirmed by $16 \mathrm{~S}$ rRNA by PCR analysis using commercial primers (forward primer 5'-AGA GTT TGA TCC TGG CTC AG$3^{\prime}$ and reverse primer 5'-AAG GAG GTG ATC CAN CCR CA-3'; Lane, 1991). Nucleotide sequence from colonies in MRS exhibited $100 \%$ homology with Bacillus licheniformis and those in EMB with Escherichia coli.

\section{Blood analysis}

Plasma was used to measure albumin, total protein, GPT, GOT, GGT, total bilirubin, direct bilirubin, glucose, total cholesterol, HDL cholesterol, BUN, creatinine and uric acid. All analyses were performed at Optifarm laboratory (Cheongwong, Chungbuk, Korea).

\section{Statistical analysis}

Pen was considered an experimental unit. All data obtained was subjected to one-way analysis of variance (SPSS Ver. 21, IBM Corp., USA). If a significant effect was observed, the difference between treatments was determined by Tukey's test. Results were expressed as treatment means with their pooled SEM. In addition, linear contrasts were used to assess the effect of $F G$ levels. A $p<0.05$ was considered statistically significant.

\section{RESULTS}

During the experimental period, none of the production-related parameters, i.e., daily body weight gain, feed intake and feed:gain ratio, were affected by the dietary treatments (Table 2 ). However, average daily gain on day 21 linearly increased $(p=0.024)$ with increasing FG levels.

On day 35, organ weights, when expressed as relative to body weight, were not affected by the dietary treatments (Table 3); however, relative bursa weight progressively declined with increasing FG levels. Jejunal villus height did not differ among dietary treatments (Table 4). Villus width linearly decreased as FG levels increased $(p=0.17)$. Jejunal crypt depth was significantly lower $(p<0.05)$ in the FG1 and FG3 groups compared with the NC group. Jejunal villus area was not affected by the FG treatments. Villus height:crypt depth ratio $(p=0.056)$ in the $\mathrm{FG}$-fed groups tended to be higher $(p=0.0558)$ compared with the NC group. Villus height:crypt depth ratio linearly increased $(p=0.0181)$ with increasing FG levels. In contrast, villus height and width, villus surface area, crypt depth and villus height:crypt depth ratio in the ileum were not 
Table 2 - Effect of fermented garlic on the growth performanceof broiler chickens ${ }^{1,2}$

\begin{tabular}{lcccccccc}
\hline Item & NC & PC & FG1 & FG3 & FG5 & SEM & P & L \\
\hline 1-21 days & & & & & & & & \\
Average daily gain, g/d/bird & 34.57 & 34.63 & 35.86 & 36.70 & 36.88 & 0.715 & 0.104 & 0.024 \\
Feed intake, g/d/bird & 55.73 & 54.57 & 55.41 & 52.63 & 56.40 & 1.597 & $>0.50$ & $>0.50$ \\
Feed:gain ratio, g/g & 1.615 & 1.574 & 1.548 & 1.435 & 1.530 & 0.042 & 0.088 & 0.078 \\
22-35 days & & & & & & & & \\
Average daily gain, g/d/bird & 70.69 & 69.22 & 68.52 & 69.43 & 66.26 & 2.577 & $>0.50$ & 0.443 \\
Feed intake, g/d/bird & 100.28 & 96.14 & 95.30 & 95.65 & 97.42 & 2.167 & $>0.50$ & $>0.50$ \\
Feed:gain ratio, g/g & 1.430 & 1.389 & 1.392 & 1.388 & 1.476 & 0.054 & $>0.50$ & $>0.50$ \\
1-35 days & & & & & & & \\
Average daily gain, g/d/bird & 49.05 & 48.95 & 49.04 & 46.93 & 48.58 & 1.566 & $>0.50$ & $>0.50$ \\
Feed intake, g/d/bird & 76.63 & 70.77 & 70.56 & 66.46 & 71.63 & 2.371 & 0.465 & $>0.50$ \\
Feed:gain ratio, g/g & 1.481 & 1.446 & 1.439 & 1.424 & 1.475 & 0.040 & $>0.50$ & $>0.50$ \\
\hline
\end{tabular}

${ }^{1} \mathrm{NC}$, negative control; PC, positive control (antibiotic at 10 ppm); FG1, fermented garlic at 0.1\%; FG3, fermented garlic at $0.3 \%$; FG5, fermented garlic at $0.5 \%$; SEM, pooled standard error of the mean; $P, p$-value analyzed by ANOVA $(p<0.05)$; L, linear effect of dietary FG.

${ }^{2}$ Mean values are presented ( $n=5$, each group).

Table 3 - Effect of fermented garlic on the relative organ weights of broiler chickens ${ }^{1,2}$

\begin{tabular}{lcccccccc}
\hline Item & NC & PC & FG1 & FG3 & FG5 & SEM & P & L \\
Liver, g/100g BW & 2.478 & 2.522 & 2.491 & 2.700 & 2.600 & 0.193 & $>0.50$ & 0.319 \\
Spleen, g/100g BW & 0.127 & 0.122 & 0.126 & 0.116 & 0.148 & 0.020 & 0.287 & 0.308 \\
Bursa of Fabricius, g/100g BW & 0.244 & 0.219 & 0.220 & 0.212 & 0.193 & 0.024 & 0.158 & 0.018 \\
Abdominal fat, g/100g BW & 1.515 & 1.690 & 1.546 & 1.797 & 1.569 & 0.187 & 0.426 & 0.440 \\
\hline
\end{tabular}

${ }^{1} \mathrm{NC}$, negative control; $\mathrm{PC}$, positive control (antibiotic at $10 \mathrm{ppm}$ ); FG1, fermented garlic at $0.1 \%$; FG3, fermented garlic at $0.3 \%$; FG5, fermented garlic at $0.5 \%$; SEM, pooled standard error of the mean; $P, p$-value analyzed by ANOVA $(p<0.05)$; L, linear effect of dietary FG.

${ }^{2}$ Mean values are presented $(n=5$, each group).

Table 4 - Effect of fermented garlic on the intestinal morphology of broiler chickens ${ }^{1,2}$

\begin{tabular}{|c|c|c|c|c|c|c|c|c|}
\hline Item & NC & PC & FG1 & FG3 & FG5 & SEM & $P$ & $\mathrm{~L}$ \\
\hline \multicolumn{9}{|l|}{ Jejunum } \\
\hline Villus height, $\mu \mathrm{m}$ & 896.1 & 827.7 & 753.7 & 734.5 & 1005.4 & 76.391 & 0.120 & 0.087 \\
\hline Villus width, $\mu \mathrm{m}$ & 130.0 & 120.7 & 116.5 & 104.2 & 92.1 & 9.217 & 0.074 & 0.001 \\
\hline Villus area, $\mathrm{mm}^{2}$ & 0.36 & 0.32 & 0.28 & 0.24 & 0.29 & 0.04 & 0.270 & 0.189 \\
\hline Crypt depth, $\mu \mathrm{m}$ & $178.6^{a}$ & $148.8^{\mathrm{ab}}$ & $108.6^{\mathrm{b}}$ & $108.3^{b}$ & $133.5^{\mathrm{ab}}$ & 16.279 & 0.033 & 0.074 \\
\hline Villus height:crypt depth ratio, $\mu \mathrm{m}: \mu \mathrm{m}$ & 5.14 & 5.78 & 7.19 & 7.18 & 7.89 & 0.668 & 0.055 & 0.018 \\
\hline \multicolumn{9}{|l|}{ lleum } \\
\hline Villus height, $\mu \mathrm{m}$ & 644.4 & 606.1 & 519.4 & 531.1 & 622.0 & 37.277 & 0.102 & 0.075 \\
\hline Villus width, $\mu \mathrm{m}$ & 147.4 & 159.1 & 153.2 & 170.5 & 152.8 & 13.725 & $>0.50$ & $>0.50$ \\
\hline Villus area, $\mathrm{mm}^{2}$ & 0.29 & 0.30 & 0.28 & 0.29 & 0.30 & 0.032 & $>0.50$ & $>0.50$ \\
\hline Crypt depth, $\mu \mathrm{m}$ & 189.5 & 177.3 & 137.7 & 143.8 & 173.8 & 20.620 & 0.356 & 0.244 \\
\hline Villus height:crypt depth ratio, $\mu \mathrm{m}: \mu \mathrm{m}$ & 3.55 & 3.74 & 3.97 & 3.90 & 3.77 & 0.506 & $>0.50$ & $>0.50$ \\
\hline
\end{tabular}

${ }^{1} \mathrm{NC}$, negative control; PC, positive control (antibiotic at $10 \mathrm{ppm}$ ); FG1, fermented garlic at $0.1 \%$; FG3, fermented garlic at $0.3 \%$; FG5, fermented garlic at $0.5 \%$; SEM, pooled standard error of the mean; $P, p$-value analyzed by ANOVA $(a, b p<0.05)$; L, linear effect of dietary FG.

${ }^{2}$ Mean values are presented ( $n=5$, each group).

affected by the dietary treatments. Neither PC or FG vs. NC affected the population of cecal microflora (Table 5). It was confirmed that the nucleotide sequence from colonies in MRS exhibited $100 \%$ homology with Bacillus licheniformis and those in EMB with Escherichia coli. None of dietary treatments affected blood parameters, such as albumin, total or direct bilirubin, GGT, uric acid, or glucose (Table 6). Creatinine, BUN, GPT, and GOT were not influenced by dietary FG compared with the NC group. Broiler chickens fed the FG5-added diet exhibited significantly higher total protein and cholesterol concentration compared with those fed the NC diet.

\section{DISCUSSION}

The present study aimed at investigating if garlic fermented by L. citreum SK2556, a novel patent strain isolated from prickled garlic product, could affect the growth performance and the intestinal morphology of 
Table 5 - Effect of fermented garlic on the cecal microflora of broiler chickens ${ }^{1,2}$

\begin{tabular}{lcccccccc}
\hline Item & NC & PC & FG1 & FG3 & FG5 & SEM & P & L \\
\hline Lactic acid bacteria, cfu/g & 10.99 & 10.53 & 10.82 & 10.53 & 10.95 & 0.139 & 0.112 & $>0.50$ \\
Escherichia coli, cfu/g & 10.82 & 10.44 & 10.79 & 10.64 & 10.53 & 0.195 & $>0.50$ & 0.350 \\
\hline
\end{tabular}

${ }^{1} \mathrm{NC}$, negative control; PC, positive control (antibiotic at 10 ppm); FG1, fermented garlic at 0.1\%; FG3, fermented garlic at 0.3\%; FG5, fermented garlic at 0.5\%; SEM, pooled standard error of the mean; $P, p$-value analyzed by ANOVA $(p<0.05)$; L, linear effect of dietary FG.

${ }^{2}$ Mean values are presented ( $n=5$, each group).

Table 6 - Effect of fermented garlic on the blood parameters of broiler chickens ${ }^{1,2}$

\begin{tabular}{lcccccccc}
\hline Item & NC & PC & FG1 & FG3 & FG5 & SEM & P & L \\
\hline Albumin, g/dl & 0.69 & 0.72 & 0.55 & 0.79 & 0.67 & 0.070 & $>0.50$ & 0.184 \\
\hline Total protein, g/dL & 4.18 & 3.89 & 4.03 & 4.13 & 6.41 & 0.439 & 0.398 & 0.007 \\
Total bilirubin, $\mathrm{mg} / \mathrm{dL}$ & 0.89 & 1.05 & 0.91 & 1.02 & 0.93 & 0.053 & $>0.50$ & 0.341 \\
\hline Direct bilirubin, $\mathrm{mg} / \mathrm{dL}$ & 0.35 & 0.43 & 0.35 & 0.39 & 0.36 & 0.022 & $>0.50$ & $>0.50$ \\
Uric acid, $\mathrm{mg} / \mathrm{dL}$ & 6.36 & 6.44 & 7.06 & 6.61 & 6.31 & 0.250 & $>0.50$ & 0.603 \\
Creatinine, $\mathrm{mg} / \mathrm{dL}$ & $0.51^{\mathrm{ab}}$ & $0.49^{\mathrm{ab}}$ & $0.47^{\mathrm{b}}$ & $0.53^{\mathrm{a}}$ & $0.53^{\mathrm{a}}$ & 0.015 & 0.021 & 0.051 \\
BUN, mg/dL & 1.94 & 1.96 & 1.61 & 2.10 & 2.26 & 0.076 & 0.093 & 0.001 \\
GPT, U/L & $35.2^{\mathrm{ab}}$ & $31.7^{\mathrm{b}}$ & $26.3^{\mathrm{b}}$ & $37.2^{\mathrm{ab}}$ & $58.9^{\mathrm{a}}$ & 5.983 & 0.006 & $<0.001$ \\
GOT, U/L & 238.2 & 195.1 & 151.7 & 267.4 & 241.3 & 19.688 & 0.342 & 0.002 \\
GGT, U/L & 43.22 & 49.73 & 39.99 & 44.15 & 45.91 & 3.882 & $>0.50$ & $>0.50$ \\
Glucose, $\mathrm{mg} / \mathrm{dL}$ & 326.9 & 316.3 & 304.4 & 318.0 & 310.7 & 5.300 & $>0.50$ & 0.181 \\
Total cholesterol, $\mathrm{mg} / \mathrm{dL}$ & 137.5 & 143.6 & 145.9 & 139.2 & 155.5 & 2.364 & 0.118 & 0.001 \\
HDL cholesterol, $\mathrm{mg} / \mathrm{dL}$ & 75.0 & 70.7 & 70.3 & 80.6 & 80.2 & 3.045 & 0.158 & 0.045 \\
\hline
\end{tabular}

${ }^{1} \mathrm{NC}$, negative control; PC, positive control (antibiotic at $10 \mathrm{ppm}$ ); FG1, fermented garlic at $0.1 \%$; FG3, fermented garlic at $0.3 \%$; FG5, fermented garlic at $0.5 \%$; SEM, pooled standard error of the mean; BUN, blood urea nitrogen; GPT, glutamic pyruvic transaminase; GOT, glutamic oxaloacetic transaminase; GGT, gamma glutamyl transferase; HDL, high-density lipoprotein; $P, p$-value analyzed by ANOVA $(a, b<<0.05)$; L, linear effect of dietary FG.

${ }^{2}$ Mean values are presented ( $n=5$, each group).

broiler chickens. Earlier, we isolated L. citreum SK2556 strain from pickled garlic food, characterized and patented due to its ability to grow and ferment in a medium containing garlic (Kim et al., 2012). In contrast with the strain used in this study, previously reported strains (i.e., L. plantarum, B. subtilis, W. koreensis, and $L$. mesenteroides) were not isolated from garlic, nor detailed information on their garlic-fermenting capacity was informed. However, earlier studies (Wang et al., 2011; Hossain \& Kim, 2014) reported that both $W$. koreensis- and L. mesenteroides-fermented garlic produced equal amounts of active metabolites, such as alliin, allylmethyltrisulfide, diallyltrisulfide, diallyldisulfide, and 3-vinyl-[4H]-1,2-dithiin.

The addition of $F G$ to the broiler diets linearly increased average weight gain between 1-21 days, which partially explains the FG-induced decrease in feed:gain ratio. The results of the present study confirm the findings of previous studies (Choi et al., 2010; Kang et al., 2010; Ao et al., 2011; Onyimonyi et al., 2012; Hossain et al., 2014, 2015) showing that neither FG or non-fermented garlic adversely affected the feed intake in broiler chickens. Thus, the underlying mechanism of the FG-induced increase in daily weight gain is likely the consequence of improvements in nutrient digestibility, especially at early ages. The latter assumption is supported by Issa \& Abo Omar (2012), who reported that dietary garlic powder at the levels of 0.2 or $0.4 \%$ increased CP and EE digestibility in broiler chickens, although they did not find any garlic-mediated increase in growth performance. It was previously reported that dietary FG significantly increased body weight gain and feed:gain ratio, without any effect on feed intake, when added at the levels of $0.2 \%$ (Hossain et al., 2014, 2015) or $1 \%$ (Kang et al., 2010)in broiler diets.

In this study, we found that dietary FG significantly reduced jejunal crypt depth compared with the NC group. The ratio of villus height:crypt depth in jejunum linearly increased with increasing FG levels. The decrease in crypt depth observed in this study may indicate that FG may reduce villus turnover rate, and therefore, that relatively less energy is used for new tissue synthesis (Abdullah et al., 2010), ultimately reducing energy requirements to maintain villus integrity. Given that villus:crypt ratio is an indicator of the digestive capacity of the small intestine (i.e., jejunum), the increased ratio observed in this study may provide the evidence that FG may play a role in digestion and absorption, as reported elsewhere (Adibmoradi et al., 2006). Therefore, the question is how dietary FG affects intestinal morphology? One 
Lee KW, Kim GH, Kim JH, Yeon JS, Cho SB, Chang BS, Kim SK
Effects of Dietary Fermented Garlic on the Growth Performance, Relative Organ Weights, Intestinal Morphology, Cecal Microflora and Serum Characteristics of Broiler Chickens plausible explanation would be the effect of FG on gut microflora. Unfortunately, neither dietary FG or in-feed antibiotic (i.e., PC) affected the cecal microflora in this study. If this holds true for the jejunum, then the next plausible explanation is the direct impact of effectors present in the FG preparation on gut function, as also proposed by Abdullah et al. (2010).

To our surprise, we found that relative weight of bursa of Fabricius was linearly declined with increasing FG levels. This is in sharp contrast to the findings of Rahimi et al. (2011), who reported that dietary garlic significantly increased the relative weight of bursa of Fabricius compared with the non-medicated NC dietfed chickens. In addition, Hossain et al. (2014, 2015) found no effect of $L$. mesenteroides-fermented garlic on the relative bursa weight of broiler chickens. At this stage, we do not know whether FG-mediated decrease in bursa weight impaired host immunity as we did not measure any immune-related parameters. Enhanced immune modulation by dietary garlic has been well established in broiler chickens (Ao et al., 2011; Kim et al., 2013) and it is considered the putative underlying mechanism in the garlic-mediated improvement of Eimeria-induced growth depression (Arczewska-Wlosek and Swiatkiewicz, 2013; Kim et al., 2013). Similarly, the enhanced host innate and/or acquired immunity by plant-derived phytochemicals is known to prevent Eimeria infection in broiler chickens (Lee et al., 2011; Kim et al., 2013). Further studies are warranted to see whether dietary FG can alter host immune response in broiler chickens or render chickens resistant or susceptible to enteric pathogens, such as Eimeria spp. or Clostridium perfringens.

Blood parameters were not different between the NC and PC groups. This was expected, as antibiotics are not absorbed in the intestine, therefore are less likely to exhibit direct systematic effect. On the other hand, the addition of FG to the diet altered various blood characteristics of the evaluated broilers. First, higher serum total cholesterol levels were determined in the FG5 vs. NC groups, which is contrary to the wellestablished hypocholesterolemic effect of garlic (Chi et al., 1982; Konjufca et al., 1997; Ao et al., 2010, 2011; Khan et al., 2012). This contradictory result may be related to the added levels of garlic or to the fat source used. Chi et al. (1982) concluded that the effective garlic level to lower cholesterol in rats is approximately $2 \%$ when diets contain either exogenous cholesterol or $15 \%$ lard as a fat sources. Ao et al. (2010) also observed the hypocholesterolemic effect of fresh garlic added at the concentration of $2 \%$ to layer diets. In another report by Ao et al. (2011), the addition of $W$. koreensis-fermented garlic at $0.2 \%$ to a broiler diet, containing soybean oil as the single fat source, significantly lowered serum cholesterol compared with the NC group. On the other hand, adding garlic at $0.3 \%$ or $0.6 \%$ to broiler diets containing approximately $2 \%$ of soybean oil did not affect serum cholesterol levels in broiler chickens (Amouzmehr et al., 2012). Whether FG at low levels in diets containing either animal fat or plant oil as a single fat ingredient would exhibit identical effect on lipid metabolism needs to be clarified. The higher total serum protein levels in FG5 compared with NC seems secondary to the increased cholesterol levels.

In conclusion, dietary FG linearly increased average daily gain during the starter phase (1-21 days) and decreased the relative weight of the bursa of Fabricius of 35-d-old broiler chickens. Jejunal, but not ileal, crypt depth was significantly lower both in the FG1 and FG2 groups compared with the NC group. Consequently, villus height:crypt depth ratio in the jejunum linearly increased as FG levels increased. Finally, dietary FG significantly increased serum total protein and cholesterol concentrations compared with the NC group. Further studies are warranted to determine of dietary FG can modulate local or systemic immune responses in naïve chicken or, preferentially, in enteric disease chicken models, and affect lipid metabolism in the presence or absence of exogenous cholesterol in the diet.

\section{ACKNOWLEDGMENTS}

This work was carried out with the support of Cooperative Research Program for Agriculture Science \& Technology Development (Project No. PJ010906), Rural Development Administration, Republic of Korea.

\section{REFERENCES}

Abdullah AY, Mahmoud KZ, Nusairat BM, Qudsieh RI. Small intestinal histology, production parameters, and meat quality as influenced by dietary supplementation of garlic (Allium sativum) in broiler chicks. Italian Journal of Animal Science 2010;9:e80.

Adibmoradi M, Navidshad B, Seifdavati J, Royan M. Effect of dietary garlic meal on histological structure of small intestine in broiler chickens. Journal of Poultry Science 2006;43:378-383.

Amouzmehr A, Dastar B, Nejad JG, Sung KI, Lohakare J, Forghani F. Effects of garlic and thyme extracts on growth performance and carcass characteristics of broiler chicks. Journal of Animal Science and Technology 2012;54:185-190.

Ao X, Yoo JS, Lee JH, Jang HD, Wang JP, Zhou TX, et al. Effects of fermented garlic powder on production performance, egg quality, blood 
Lee KW, Kim GH, Kim JH, Yeon JS, Cho SB, Chang BS, Kim SK profiles and fatty acids composition of egg yolk in laying hens. Asian Australasian Journal of Animal Sciences 2010;23:786-791.

Ao X, Yoo JS, Zhou TX, Wang JP, Meng QW, Yan L, et al. Effects of fermented garlic powder supplementation on growth performance, blood profiles and breast meat quality in broilers. Livestock Science 2011;141:85-89.

Chi MS, Koh ET, Stewart TJ. Effects of garlic on lipid metabolism in rats fed cholesterol or lard. Journal of Nutrition 1982;112:241-248.

Choi IH, Park WY, Kim YJ. Effects of dietary garlic powder and $\alpha$-tocopherol supplementation on performance, serum cholesterol levels, and meat quality of chicken. Poultry Science 2010;89:1724-1731.

Chun SM, Kim SC, Joo ST, Yang HS. Effect of dietary garlic-husk probiotics on laying hens performance and egg quality. Journal of Agriculture and Life Science 2015;49:137-146.

Ham JS, Lee SG, Kim MK, Oh MH, Jeong SG, Kim DH, et al. Inhibitory activity of garlic fermented by Pediococcus pentosaceus KACC 91419 against antibiotic-resistant pathogens. Asian Australasian Journal of Animal Science 2010;23:1236-1243.

Hossain MM, Lee SI, Kim IH. Corrigendum to Effect of dietary Korean aged garlic extract by Leukonostoc mesenteroides KCCM35046on production, hematological status, meat quality, relative organ weight, targeted Escherichia coli colony and excreta gas emission in broilers. Animal Feed Science and Technology 2015;206:126.

Issa KJ, Abo Omar JM. Effect of garlic powder on performance and lipid profile of broilers. Open Journal of Animal Science 2012;2:62-68.

Kang $\mathrm{HH}$, Kim $\mathrm{CH}$. Effects of dietary supplementation with rice bran oil on the growth performance, blood parameters, and immune response of broiler chickens. Journal of Animal Science and Technology 2016;58:12.

Kang HK, Seo OS, Choi HC, Chae HS, Na JC, Bang HT, et al. Effect of dietary supplementation of fermented by-products of garlic and onion on production performance, blood components and cecal microflora in broiler chickens. Korean Journal of Poultry Science 2010;37:433-438.

Khan RU, Nikousefat Z, Tufarelli V, Naz S, Javdani M, Laudadio V. Garlic (Allium sativum) supplementation in poultry diets: effect on production and physiology. World's Poultry Science Journal 2012;68:417-424.

Kim DK, Lillehoj HS, Lee SH, Lillehoj EP, Bravo D. Improved resistance to Eimeria acervulina infection in chickens due to dietary supplementation with garlic metabolites. British Journal of Nutrition 2013;109:76-88.

Kim SJ, Kwon CH, Park BC, Lee CY, Han JH. Effects of a lipid-encapsulated zinc oxide dietary supplement, on growth parameters and intestinal morphology in weanling pigs artificially infected with enterotoxigenic Escherichia coli. Journal of Animal Science and Technology 2015;57:4.
Effects of Dietary Fermented Garlic on the Growth Performance, Relative Organ Weights, Intestinal Morphology, Cecal Microflora and Serum Characteristics of Broiler Chickens

Kim SK, Cho SB, Kim SO, Park JW. Novel Leuconostoc citreum enhancing antioxidant activity by garlic fermentation [Korea Patent 10-1159237]. Gwangjin-gu, Seoul, Koreia: Konkuk University and Chodae; 2012.

Konjufca VH, Pesti GM, Bakalli RI. Modulation of cholesterol levels in broiler meat by dietary garlic and copper. Poultry Science 1997;76:1264-1271.

Lane DJ. 16S/23S rRNA sequencing. In: Stackebrandt E, Goodfellow M, editor. Nucleic acid techniques in bacterial systematics. New York: John Wiley \& Sons; 1991. p.115-176.

Lee SH, Lillehoj HS, Jang SI, Lee KW, Park MS, Bravo D, et al. Cinnamaldehyde enhances in vitro parameters of immunity and reduces in vivo infection against avian coccidiosis. British Journal of Nutrition 2011;106:862869.

Onyimonyi AE, Chukwuma PC, Chinenye I. Growth and hypocholesterolemic properties of dry garlic powder (Allium sativum) on broilers. African Journal of Biotechnology 2012;11:2666-2671.

Rahimi S, Zadeh ZT, Torshizi MAK, Omidbaigi R, Rokni H. Effect of the three herbal extracts on growth performance, immune system, blood factors and intestinal selected bacterial population in broiler chickens. Journal of Agricultural Science and Technology 2011;13:527-539.

Sakamoto K, Hirose H, Onizuka A, Hayashi M, Futamura N, Kawamura Y, et al. Quantitative study of changes in intestinal morphology and mucus gel on total parenteral nutrition in rats. Journal of Surgical Research 2000;94:99-106.

Wang JP, Yoo JS, Jang HD, Cho JH, Kim IH. Effect of dietary fermented garlic by Weissella koreensis powder on growth performance, blood characteristics, and immune response of growing pigs challenged with Escherichia coli lipopolysaccharide. Journal of Animal Science 2011;89:2123-2131.

Wilson EA, Demming-Adams B. Antioxidant, anti-inflammatory, and antimicrobial properties of garlic and onion. Nutrition and Food Science 2007;37:178-183.

Yan L, Ming QW, Kim IH. Effects of fermented garlic powder supplementation on growth performance, nutrient digestibility, blood characteristics and meat quality in growing-finishing pigs. Animal Science Journal 2012;83:411-417.

Yang L, Kim IH. Effects of dietary supplementation of fermented garlic powder on growth performance, apparent total tract digestibility, blood characteristics and faecal microbial concentration in weanling pigs. Journal of Animal Physiology and Animal Nutrition 2013;97:457464. 
